\title{
Pastoralinstruktion und katholische Berufsorganisationen
}

\author{
von Jesús Iribarren
}

Die Beziehungen zwischen den katholischen Berufsorganisationen für Publizistik und den kirchlichen Hauptstellen für die sozialen Kommunikationsmittel wurden bereits mehrfach behandelt. ${ }^{1}$ Die neu erschienene Pastoralinstruktion "Communio et Progressio" liefert jetzt entscheidende Elemente zur Lösung der Unklarheiten, Kontroversen und Pressionen, die offensichtlich trotz allem in verschiedenen Teilen der Welt weiterhin bestehen. Keinem der in den neutralen Medien beruflich Engagierten, aber auch den offiziell katholischen Publikationen kann der (Un)Abhängigkeitsgrad von der kirchlichen Hierarchie nicht gleichgültig sein. Deswegen seien hier auf Grund der neuen Pastoralinstruktion einige klärende Hinweise am Beispiel der Katholischen Weltunion der Presse (UCIP) versucht.

\section{Die freien Vereinigungen und ibr Wirkungsfeld}

Eine Kirche, die in ihrer ganzen Soziallehre die Vereinigungsfreiheit zu rechtmäßigen Zwecken und die subsidiäre Rolle der staatlichen Einmischung in staatsbürgerliche Initiativen unterstreicht, kann nicht versäumen, dieselben Grundsätze auf das Gebiet der sozialen Kommunikationsmittel anzuwenden. Tatsächlich verteidigt „Communio et Progressio" das Prinzip freier Vereinigungen für Berufskräfte und Verbraucher.

Die Instruktion tut dies (ehe sie zur konfessionellen Ebene übergeht), indem sie allgemein von den "Medien“ als von den Förderern des menschlichen Fortschritts spricht (II, 2, 2). Die aus Katholiken zusammengesetzten Vereinigungen sind also in dieser prä-konfessionellen Abteilung inbegriffen. Nr. 79 vermerkt unter den Zielsetzungen der Vereinigungen von Kommunikatoren, deren Existenz eben dadurch Rechtmäßigkeit und Empfehlung erhält, "die Schwierigkeiten ihres Berufes zu meistern“. Als eine der möglichen - aber nicht der ausschließlichen, da eine derartige Ausschließlichkeit durch nichts auferlegt wird - Aufgaben der Berufsvereinigungen setzt dieselbe Nr. 79 hinzu, daß sie sich „eine Berufsordnung geben können, um gemeinsame Probleme und Aufgaben im Sinne der umfassenden Ziele sozialer Kommunikation zu lösen".

Der von der Instruktion vorgesehene Rahmen geht weit über eine „deontologische Regelung " im strengen Sinne hinaus, ${ }^{2}$ weil es sich dabei um eine bessere Anpassung des Mediengebrauchs „ad commodius ministrandum hominibus“, d. h. zu einem besseren Dienste am Menschen, handelt. Es darf nicht vergessen werden, daß dies das allgemeine Thema des Kapitels ist.

Auf dem Gebiet der Vereinigungen, die spontan aus gesellschaftlichen Gegebenheiten entstehen, wird in Nr. 83 die Zweckmäßigkeit von "Rezipienten-Vereinigungen“" unterstrichen oder dazu angeregt, die Hilfe anderer Organisationen in Anspruch zu nehmen, die unter anderen, weiter gesteckten Zielen auch solche miteinschließen, die diesen Rezipienten-Vereinigungen eigen sind.

Msgr. Jesús Iribarren war als Nachfolger von Edule Gabel $(\uparrow 1968)$ bis zum Dezember 1971 Generalsekretär der "Union Catholique Internationale de la Presse " (UCIP) in Paris. Bis zur Regelung seiner Nachfolge bleibt er Kommissarischer Generalsekretär der UCIP. 
Die Vereinigungen finden in der Instruktion eine weitere Rechtfertigung, wenn von den Pflichten der Regierenden und der Regierten und einer Zusammenarbeit zwischen beiden die Rede ist (II, 2,3). Die Kommunikation ist nicht allein Aufgabe des Staates, sondern der ganzen Gesellschaft. „Alle sind verpflichtet, die Freiheit zu verteidigen ... (84). Die politische Gesellschaft hat auf diesem Gebiet ihre Verantwortungen zu übernehmen, und dies gilt sowohl für die Einzelnen als auch für die Gruppen (85). „Es ist nützlich und oft auch notwendig, daß zu diesem $Z$ weck Vereinigungen gebildet werden ${ }^{*}(85)$.

Die Träger staatlicher Gewalt haben ihrerseits, durch eine entsprechende Gesetzgebung, die Ausdrucksfreiheit, das Informationsrecht und die Religionsfreiheit zu schützen. Manchmal aber kommen die Hindernisse von der Staatsgewalt selbst, vom Drudk wirtschaftlicher Gruppen, vom Staatsmonopol. Die Staatsgewalt kann nicht alleiniger Schiedsrichter der Lage sein. Daher „muß den Staatsbürgern durch Gesetz eine volle Kontrollmöglichkeit der gesamten Verwaltung von Kommunikationsmitteln garantiert werden " (87). Diese Kontrolle kann natürlich nicht in Spannungsmomenten mit der Gewalt von isolierten Staatsbürgern wirksam ausgeübt werden. Hier liegt eine Aufgabe für die Vereinigungen von Kommunikatoren und Rezipienten, von denen eben die Rede war.

Nr. 88 bezieht sich insbesondere auf die Informatoren, die eben im Hinblick auf die Zusammenarbeit mit den Behörden und um mögliche Spannungen zu überwinden, „von sich aus Gremien mit eigenen Satzungen schaffen müssen; diese sollen in allen hier auftauchenden Sachfragen beratend tätig werden. In diese Gremien sollen Vertreter aller Gruppen der Gesellschaft berufen werden. So kann man hoffen, daß staatliche Eingriffe und ein Obergewicht wirtschaftlicher Interessen vermieden werden " (also Verteidigung der Berufsvereinigungen gegen den Druck von Gruppen). "Andererseits könnte dadurch die Zusammenarbeit unter den Kommunikatoren so verbessert werden, daß die Gesamtanstrengung der Medien für das Gemeinwohl wirksamer wird."

Dessen ungeachtet werden "vielleicht ${ }^{\star}$, „hier und $\mathrm{da}^{\text {“ ("fortasse }}$, „in aliquibus locis") die öffentlichen Behörden selbst Kontrollgremien schaffen müssen. Ein gefährliches Gebiet; daher eine Alarmstimme: der Meinungspluralismus muß gewahrt werden. Diese eventuell von den Behörden geschaffenen Gremien müssen derart zusammengesetzt sein, daß "die repräsentative Vertretung der verschiedenen gesellschaftlichen Gruppierungen gesetzlich geordnet ist" (88).

Nach dem bisher Gesagten geht es also darum, dem Dienste der Medien am Gesamtfortschritt des Menschen die volle Effizienz zu geben. An diesem Dienste - ohne Einschränkung der Tätigkeitsgebiete - können die Vereinigungen von Rezipienten (Staatsbürger jedwelchen Landes, jedwelcher Religion und jedwelchen ideologischen Systems) und von Kommunikatoren (unter der alleinigen Bedingung, daß es sich um verantwortliche Menschen handelt, die sich ihrer Pflichten im Dienste an der Menschheit bewußt sind) mitwirken. Die Pastoralinstruktion ist weder restriktiv, noch kann sie es sein.

Zielgruppe: alle, die diesen Beruf angehen, dessen Ausübung ein Faktor des menschlichen Fortschritts sein kann. In besonderer Weise werden die Uberwindung der beruflichen Schwierigkeiten, die Schaffung einer Berufsordnung von innen her, die Vermeidung einer Einmischung gewisser Gruppierungen durch Druckmaßnahmen, der gemeinsame und koordinierte Dienst am Gemeinwohl vermerkt. 
Aufgrund der Einfügung dieser Aussagen in das 2. Kapitel des 2. Teils der Instruktion, das einen absolut allgemeinen Charakter aufweist, ist es offensichtlich, daß die theoretisch vorgesehenen Vereinigungen in ihrer praktischen Durchführung in religiöser Hinsicht neutral, interkonfessionell, de facto konfessionell - ohne daß dies eine offizielle Anerkennung erfordert - oder offiziell konfessionell sein können. Die eine oder andere Wesensart wird allein von den historischen, soziologischen und rechtlichen Bedingungen jedes Landes abhängig sein. ${ }^{3}$

Allen Pressevereinigungen zeigt die Kirche ihre Sympathie. Sie kann sich nur darüber freuen, daß es in diesen Vereinigungen Katholiken gibt; je mehr, desto besser. Es wären dies "Katholiken in der Presse" nach der Leitlinie, die die UCIP in Zukunft immer stärker betonen will. Die mittelbare oder unmittelbare pastorale Besorgnis, die die Hierarchie in dieser Welt der Pressevereinigungen durch die verschiedenen seelsorglichen Einrichtungen der Hierarchie walten lassen kann, wird zum Teil von Gewicht und Zahl der in solchen neutralen, interkonfessionellen oder katholischen aber nicht-konfessionellen Vereinigungen anwesenden katholischen Berufskräften, und zum Teil vom Wohlwollen der nicht-katholischen Berufskräfte abhängen. Diese Besorgnis wird natürlich durch einen animierenden und nicht durch einen direktiven Charakter gekennzeichnet sein, wie es "Communio et Progressio" etwas später (104-105) betont, indem erklärt wird, die Kirche sei „daran interessiert, mit den Kommunikatoren, gleich welcher religiösen Überzeugung, ins Gespräch zu kommen" (105).

Die ganze in den Nummern 73 bis 100 dargelegte Lehre (Möglichkeiten und Pflichten der Kommunikatoren und Rezipienten; Zusammenarbeit) ist auf Grund ihrer allgemeinen Problemstellung sehr wohl auf Vereinigungen wie die UCIP anzuwenden, den Treffpunkt von Berufsinformatoren, vor 40 Jahren spontan entstanden und mit allen von der Instruktion bisher angegebenen Merkmalen versehen. Diese Allgemeinheit der Problemstellung wird durch den Umstand, daß die Vereinigungen katholischer Berufskräfte sich vornehmen, wie ein christliches Ferment auf diesem Gebiet zu wirken, sofern zuweilen isolierte katholische Journalisten und zuweilen ganze nationale oder regionale Pressevereinigungen einverleibt werden, nicht beeinträchtigt. Die Tatsache, daß eine gewisse Tätigkeit mit einem katholischen Bewußtsein ausgeübt wird, behindert nicht ihre fördernde Eigenschaft am menschlichen Fortschritt, sondern kennzeichnet dieselbe.

\section{Die Kirche kann nicht uninteressiert sein}

Gäbe es für die katholischen Informatoren und ihre Vereinigungen eine Zweiteilung zwischen Beruf und Glaube, dann gäbe es das Problem nicht.

Nun ist es aber so, daß die "Gläubigen“, aus der einfachen Tatsache heraus, daß sie Gläubige sind, einem Magisterium und einer Disziplin unterworfen sind; und daß gewisse Vereinigungen als "katholisch" bezeichnet werden wollen, womit sie selbst eine gewisse Art der Verbindung mit der kirchlichen Autorität verlangen. Bedeutet dies nicht, daß die Journalisten und ihre Gremien eine willentliche Abhängigkeit schaffen, die die ursprüngliche Vereinigungsfreiheit beeinträchtigt (in derselben Weise wie in der staatlichen Gesellschaft die Vereinigungsfreiheit sich gewissen geregelten Normen unterwerfen muß)? Ohne Zweifel. 
Andererseits haben die Mitglieder des „Berufsapostolats“ mit jedem Tage mehr Zugang zur Feder und zur Wortmeldung, so daß sie nach und nach in die Welt der "Medienfachlkräfte" Eingang finden. Werden solcherart die Grenzen nicht verwischt? In gewissem Sinne sicherlich. Kann dies aber irgendwelche Unklarheit auf dem Gebiete der Strukturen bewirken? Belehrung und Licht darüber werden uns schichtenweise im 3. Teil von "Communio et Progressio" erteilt, wenn die besondere Tätigkeit der Katholiken in den Massenmedien angeschnitten wird. Dies betrifft Journalisten, die in der Ausübung ihres Berufes Katholiken sind.

\section{a) Die katholischen Berufskräfte}

Unterstrichen werden ihre erstrangige Aufgabe, ihre Beteiligung am Auftrag, den die Christen in der Welt zu erfüllen haben, „das wichtige Zeugnis, das sie in religiös neutralen Redaktionen und Institutionen als Christen ablegen“ (103). Als Begleiterscheinung dieser Bedeutung wird gesagt, daß es „ihr Recht ist, von der Kirche geistliche Hilfe zu erhalten" (104).

Kein einziges Wort findet man aber über eine organisatorische „Umrahmung“, oder über irgendwelche Art einer strukturellen Verbindung mit seelsorglichen Einrichtungen.

b) Die Teilhaber des Berufsapostolats im Gebrauch der Massenmedien

„Diejenigen, die irgendwie die Stimme der Kirche repräsentieren, werden immer häufiger aufgefordert, für Presse, Hörfunk, Fernsehen und Film Beiträge zu leisten ..." (106).

Die Hierarchie kann diese ihr aufgetragene Verantwortung nicht leichtfertig übergehen: sie hat sich darauf vorzubereiten. „Es ist Aufgabe der kirchlichen Hauptstellen und anderer fachlicher Einrichtungen, dafür zu sorgen, daß derzeitige und künftige Mitarbeiter für die Medien sorgfältig ausgebildet und rechtzeitig vorbereitet werden“ (106). Es handelt sich hier nicht hauptsächlich um eine technische Ausbildung (die ebenfalls in verschiedenem Maße notwendig ist und die für die fortgeschritteneren Grade einer anderen Art von Ausbildungszentren obliegt), sondern um eine geistige Ausbildung, die den Schülern in katholischen Schulen und Einrichtungen gegeben wird (107), um die in den Seminaren gegebene moral- und pastoraltheologische Ausbildung (108), um die Förderung von Berufen in der sozialen Kommunikation, namentlich in den Entwicklungsländern (109), da dort zu einem großen Teil die Zukunft der Kirche liegt. „Bischöfe, Priester, Ordensleute und Laienverbände müssen entsprechend ihrer jeweiligen Verantwortung zu einer Medienerziehung aus christlicher Sicht beitragen. Dabei darf der gesamtgesellschaftliche Bezug nicht fehlen " (110). In derselben Nr. 110 wird „eine gewisse Vertrautheit und ein direkter Umgang mit den Kommunikationsmitteln" empfohlen, sowie "ein Austausch von Erfahrungen und ein gründliches Nachforschen mit den Kommunikatoren".

Erneut drängt Nr. 111 darauf, daß in den Priesterseminaren eine theoretische und gebrauchstechnische Ausbildung in den Medien erteilt wird, „eine Voraussetzung, ohne die überhaupt niemand mehr apostolisch tätig“ in der Welt von heute sein kann.

Wie man sieht, ist "Communio et Progressio" darum besorgt, daß diejenigen, die am modernen Apostolat beteiligt sind, den notwendigen Vorbereitungsgrad für den 
Pastoralgebrauch der Massenmedien erlangen. Es ist eindeutig klar, daß diese Ausbildung und die Zentren, in welchen sie erteilt wird, voll und ganz in das Feld der Pastoralstellen und der kirchlichen Hierarchie fallen. ${ }^{4}$

Von der Präsenz der Katholiken (Berufskräfte der "Medien“ und Berufskräfte des Apostolats) in allen Medien zum besonderen Medium "Presse“ übergehend (Nr. 136 bis 141), unterscheidet die Instruktion - ohne eine Systematisierung direkt vorzuschlagen $-z$ wischen verschiedenen Stufen des Engagements der katholischen Berufskräfte mit der kirchlichen Hierarchie.

Diejenigen Ubersetzungen, die diese Nummern auf die „katholische Presse ${ }^{“}$ oder „auf jene, die im Namen der Kirche sprechen " beschränken, sind dem Originaltext nicht treu. Im Original ist stets die Rede von den "Katholiken in der Presse“: „journalistische Tätigkeit der Katholiken“ ("litteraria Catholicorum navitas", "Catholicorum scriptorum opera “ - 137-138), „von Katholiken geleitete Nachrichtenagenturen" (139). Einen Schritt weitergehend empfiehlt Nr. 140 die Lektüre der „katholischen Presse"; an zwei verschiedenen Stellen (141) wird ein Unterschied gemacht zwischen den Publikationen, in welchen die Katholiken versuchen, auf die Probleme des aktuellen Geschehens „eine Antwort im Sinne des kirchlichen Lehramtes zu geben " (jedoch in der Achtung der Meinungsvielfalt) und den "katholischen Zeitungen, die als offizielle Organe der Kirche gelten" (in welchen ebenfalls, gemeinsam mit der Meinung der Einrichtung, deren offizielles Organ sie sind, Platz für die freie Meinungsäußerung der Leser in Fragen, die der Diskussion offenstehen, eingeräumt wird).

\section{Berufsvereinigungen und pastorale Stellen}

Wie man sieht, hat die Instruktion bisher keinerlei Andeutung auf eine organische Beziehung der Abhängigkeit, welcher Art sie auch sei, gemacht. Sie beschränkt sich darauf, die Fakten und die Lehre darzulegen:

a) Berechtigung und Opportunität von Berufsvereinigungen der Massenmedien;

b) Bedeutung der katholischen Berufskräfte und ihr Recht, geistliche Hilfe von der Kirche $z u$ erhalten;

c) immer stärkerer Zugang zu den Massenmedien „derjenigen, die auf die eine oder die andere Art die Kirche vertreten" und Opportunität einer angepaßten Vorbereitung, miteinbegriffen in den Seminaren;

d) vielfältige Formen der Präsenz von Katholiken in der Presse: journalistische Tätigkeit, Pressemitarbeit, Agenturen ...

e) verschiedene Grade des Engagements der katholischen Presse: Presse von Katholiken, Presse als offizielles Organ der Tätigkeit und Einrichtungen der Kirche.

In Kapitel 4 von "Communio et Progressio “ wird das Thema "Einrichtungen, Mitarbeiter, Organisation" angeschnitten. Es darf nicht außer acht gelassen werden, daß die Instruktion komplementär zum Konzil ist und aus der pastoralen Perspektive geschrieben wurde. Deshalb sollte man im voraus vermuten, daß die päpstliche Kommission nicht gesetzgebend einwirken will, auf die nicht-katholischen Berufsorganisationen, auf solche, die Katholiken und Nicht-Katholiken vereinen, auf die Tätigkeit der katholischen Berufskräfte in neutralen bzw. in katholischen Medien, sofern sich diese Tätigkeit auf die technischen Aspekte oder das allgemeine Wirken 
der Massenmedien als Faktoren des menschlichen Fortschritts - wovon der ganze zweite Teil spricht - beschränkt.

Tatsächlich setzt die Pastoralinstruktion diese Tätigkeit und diese Vereinigungen mit ihrer eigenen Persönlichkeit, ihren eigenen Satzungen, ihrer besonderen Aufgabe voraus, und zwar in einer den von der Hierarchie gegründeten und abhängigen Vereinigungen nahestehenden, aber von ihnen verschiedenen Linie.

Das apostolische Gebiet ist es, das in der Instruktion organisiert und strukturiert wird. Wir wollen hier stufenweise vorgehen: Die apostolische Fruchtbarkeit der Medien ist eine derart wichtige Angelegenheit, daß sie das Gebet und den persönlichen Einsatz aller Gläubigen erfordert (163).

Hier fehlt etwas anderes: bei den Verantwortlichen und den Realisatoren eine apostolische Mentalität zu schaffen und fähige Menschen den zu der sozialen Kommunikation in Beziehung stehenden Organisationen einzuverleiben. Natürlich auch, eine in diesem Sinne ausgerichtete Ausbildung, sei es für Laien oder Geistliche (164).

Handelt es sich um pastorale Bemühungen, so obliegt die ganze Planung und Koordinierung (aus ihrem Wesen selbst heraus) den Hirten: jedem Bischof in seiner Diözese, einer Kommission oder einem beauftragten Bischof für den nationalen Bereich, der Päpstlichen Kommission für die ganze Kirche (165).

In guter Theologie ist jeder Katholik ein Apostel, kraft eines "Auftrags“, den er aufgrund seiner Taufe erhielt. Die Apostolatsformen können sehr unterschiedlich sein. Der kirchlichen Leitung obliegt es, sie anzuregen und zu koordinieren. Sie soll die Katholiken und ihre Verbände zu freier und spontaner Initiative ermutigen. Nur in zwei Fällen soll sie sich die Leitung einer konkreten Unternehmung vorbehalten (was etwas anderes ist als eine allgemeine Koordination): wenn sie ihrem Wesen nach in den Bereich des priesterlichen Dienstes gehört, oder wenn die Leitung im Interesse der Gläubigen von der Hierarchie getragen werden muß (166).

Es ist selbstverständlich, daß, um ihren Auftrag auf einem so speziellen Gebiet wie dem der Massenmedien ausführen zu können, die Bischöfe sich dabei von Geistlichen und Laien beraten lassen müssen. Hier tritt die Rolle der diözesanen oder überdiözesanen Stellen (168) sowie der nationalen Hauptstellen (169) in Erscheinung. Die Aufgaben, die in ihren Bereich fallen, sind außer den Anliegen ihrer eigenen Strukturierung und ihres internen Lebens, allesamt seelsorglicher Art: Vorlage der Haushaltspläne für ihren eigenen Wirkungsbereich (167), Vorbereitung des „Welttags der Kommunikationsmittel“ $(167,168)$, pastorale Koordinierung des apostolischen Wirkens auf dem Gebiet der sozialen Kommunikation (168 und 170) und, in besonderer Art, Erziehung für Geistliche und Laien (170) und sachgerechte Vorbereitung religiöser Filme und Sendungen (170). Zu ihrem Aufgabenbereich gehört es, allgemeine Richtlinien für die apostolischen Bemühungen zu erlassen (172), und dies was auch logisch ist - in Verbindung mit den Bischofskommissionen anderer Länder und der Päpstlichen Kommission: also waagerechte und senkrechte Verbindung (172). $\mathrm{Da}$ sie kirchliche Einrichtungen sind, ist vorzusehen, daß eben die Kirche sie damit beauftragen wird, ihr Sprachrohr zu sein und sich um ihre Offentlichkeitsarbeit zu bemühen (174-176). Die Ordensgemeinschaften werden in ihren Bemühungen mit den diözesanen, regionalen, nationalen, kontinentalen Stellen zusammenarbeiten (177).

Die Unterscheidung zwischen den strukturell von der Hierarchie abhängigen Organisationen, die Organisationen "der" Kirche sind, und den Verbänden katholischer Berufskräfte, die „in“ der Kirche bestehen, könnte nicht deutlicher sein. Als ob die 
Seite um Seite benützte Sprache nicht klar genug wäre, wird noch die Beziehung zwischen beiden Kategorien des näheren präzisiert.

Die kirchlichen Einrichtungen (gleich welcher Stufe) werden

1. - die Hilfe der Kommunikatoren und ihrer Vereinigungen beanspruchen (,hominum artes communicationis pro munere exercentium atque eorum sodalitiorum") (171).

2. - "ihnen Unterlagen und verbindliche Beratung geben und ihnen die geistliche Hilfe anbieten, deren sie bedürfen“ („documenta, monita, adiumenta pastoralia quibus forsitan indigeant") (171).

Auf nationaler und internationaler Ebene werden die nationalen Hauptstellen des Episkopats und die zentralen Stellen der Ordensgemeinschaften den internationalen Organisationen UCIP, OCIC und UNDA im Rahmen deren Satzungen, die vom Apostolischen Stuhl gebilligt wurden", Hilfe leisten (adiutricem operam praebebunt $\left.{ }^{\alpha}\right)(178)$.

Gleichzeitig mit der Anerkennung der Persönlichkeit dieser drei Organisationen weist die Instruktion in Nr. 179 auf zahlreiche Zielsetzungen beruflicher Art hin, die sich neben den apostolischen Bemühungen bieten (wir beschränken uns hier auf die UCIP): Förderung der nationalen katholischen Berufsorganisationen, Erforschung und Entwicklung der Kommunikationsmittel, Schärfung des Solidaritätsbewußtseins und Stärkung des internationalen Austauschs, Förderung der Präsenz von Katholiken in den Massenmedien, mit der angepaßten Koordinierung der Projekte, die auf diesem Gebiet und auf internationaler Ebene bestehen, neue Initiativen - besonders in den Entwicklungsländern -- Förderung von Publikationen, die dem Fortschritt der menschlichen Gemeinschaft und dem Leben des Gottesvolkes dienen.

Die Instruktion hat Beispiele angegeben: sie hat die Liste der Tätigkeiten, die etwa der UCIP eigen sind, weder zu bestimmen noch auszuschöpfen. ${ }^{5}$ Den Berufsorganisationen und nicht der Instruktion fällt es zu, solche Programme aufzustellen. Es genügt, daß gesagt wird, die pastoralen Stellen würden die Hilfe etwa der UCIP beanspruchen (171), und uns selbst helfen (178). Ein neuer, indirekter Beweis der Persönlidhkeit, welche die Instruktion den Berufsorganisationen zuerkennt, ist, daß sie die UCIP, OCIC und UNDA auffordert, „untereinander zusammenzuarbeiten, um die Probleme, die sich ihnen gemeinsam stellen, auch gemeinsam aufzugreifen und $\mathrm{zu}$ lösen" (179).

Die Instruktion ist bis zum Schluß realistisch: der Dienst, den die katholischen internationalen Organisationen für soziale Kommunikationsmittel der Kirche leisten, ist so wichtig, daß die Hierarchie die nationalen Hauptstellen der Kirche und die nationalen Berufsorganisationen zu deren Unterstützung, sogar finanzieller Art, einlädt. „Mit aktiver Unterstützung durch die Hauptstellen werden die Bischofskonferenzen und die katholischen Berufsverbände in Bereich der sozialen Kommunikation den internationalen Vereinigungen jene finanziellen Mittel und Hilfen zu Verfügung stellen, die notwendig sind, damit diese ihr Ziel erreichen " (180). Alle Übersetzungen sind in dieser Beziehung sehr deutlich. ${ }^{6}$

Zusammengefaßt machen die Aussagen der Pastoralinstruktion für eine Berufsorganisation wie etwa die UCIP klar:

1. Die UCIP will eine internationale "katholische" Organisation sein, und sie weiß, daß - genau wie für alle anderen internationalen katholischen Organisationen - 
diese offizielle Anerkennung vonseiten des Heiligen Stuhls gewisse Bedingungen und Modalitäten mit sich bringt, deren konkrete Formen gegenwärtig untersucht werden. Die kompetenten Organe für diese Anerkennung sind das Staatssekretariat und der Laienrat.

2. In ihrer Eigenschaft als Berufsorganisation, die Presseeinrichtungen und -leute vereinigt, besitzt die UCIP ihre eigene Persönlichkeit und Organisationsautonomie, die ihr zuerkannt wird.

3. Der kirchlichen Hierarchie obliegt die Koordinierung des ganzen apostolischen Wirkens. Auf dem Gebiet der Massenmedien wurde eine solche Aufgabe der Päpstlichen Kommission für Kommunikationsmittel den bischöflichen Nationalstellen und den von der Hierarchie abhängenden nationalen oder diözesanen Stellen zugeschrieben. Die UCIP, ihre vier internationalen Föderationen und deren nationale Mitglieder sind $\mathrm{zu}$ engen Kontakten und zur Zusammenarbeit in den jeweiligen Bereichen bereit.

4. Es wäre keine angepaßte Form dieser Zusammenarbeit, vorzumerken, daß die Einrichtungen seelsorglicher Art solchen beruflicher Art beitreten könnten oder umgekehrt. Keinerlei Abhängigkeitsbeziehung soll zwischen beiden Kategorien bestehen, in keiner der beiden Richtungen und auf keiner Stufe.

5. Nichts verhindert, daß Personen, die tatsächlich die Information als Beruf ausüben, selbst wenn sie zugleich einer Pastoralstelle angehören, daß Informationsdienste und Nachrichtenbulletins kirchlicher Einrichtungen, Tageszeitungen oder Zeitschriften, die von Diözesen oder Ordensgemeinschaften herausgegeben werden, Lehrkräfte, Forscher und Journalistenschulen, die ihre Tätigkeit auf dem pastoralen Sektor ausüben, (aufgrund dieser beruflichen Aspekte) den entsprechenden nationalen Verbänden beitreten und mit ihnen - durch eine der vier Föderationen - der UCIP als Dachorganisation.

In zahlreichen Fällen wird der Beitritt von Personen und Publikationen zur vollberechtigten Mitgliedschaft ermöglicht, wie dies übrigens häufig geschieht. Es ist z. B. der Fall der fast vollzähligen Bistumspresse. In anderen Fällen - je nach Wesensart oder Periodizität gewisser Publikationen oder gewisser Nachrichtenbulletins - ist in den Föderationen eine Kategorie assoziierter Mitglieder vorgesehen, die eingeschränkte Rechte und Pflichten umfaßt; dieselben sind in den jeweiligen Satzungen angegeben.

Indem sie der einen oder anderen Kategorie beitreten, bringen die Journalisten nicht die Persönlichkeit der Pastoraleinrichtungen mit sich, noch besteht Anlaß zu jedwelcher Verwechslung. Eine Verwechslung könnte nur im Falle eines Beitritts von Leitern pastoraler Stellen als solchen entstehen, oder wenn sie in Wirklichkeit keine deutlich sichtbare berufliche Funktion in der Presse ausüben. Wir haben den aufrichtigen Wunsch, daß nicht auf eine solche Form der Mitgliedschaft oder der Verbindung gedrängt wird: wir gehören als Menschen und Einrichtungen zur Presse.

6. Die Bedeutung, die die Kirche den internationalen katholischen Medienorganisationen beimißt, ist derart, daß eine der Zusammenarbeitsformen, die die Kirche den Pastoralstellen vorschreibt, darin besteht, die wirtschaftliche Grundlage zu erleichtern, die notwendig ist, damit diese Organisationen ihren Auftrag erfüllen. Sie können, noch sollen sie für diese wirtschaftliche Unterstützung die Forderung nach der Zugehörigkeit zur UCIP stellen. 
7. Es gibt aber eine andere Mitarbeit, die vielleicht noch wichtiger als die wirtschaftliche ist, und die die Pastoralstellen der ganzen Welt der UCIP schenken können. Nämlich ihr dabei behilflich zu sein, sich fest einzupflanzen. Zehntausende katholischer Informatoren werden niemals direkt von jedwelcher kirchlichen Stelle erreicht werden. Die einzige Möglichkeit für die Präsenz der Kirche in breiten Sektoren alter und junger Nationen ist vielleicht die UCIP.

\section{Anmerkungen:}

1. Vgl. u. a.: Les Journalistes Catholistes et la hierarchie ecclésiastique in: „Journalistes Catholiques" 18:1970, Nr. 49 (Janvier-Février), S. 4-6. Jesús Iribarren: Pressefreiheit in der Kirche, oder: Marginalien zur Deontologie der katholischen Journalisten in: "Communicatio Socialis", $4: 1971,11-27$.

2. Spanische Ubersetzung "normas o cbdigos", englische „,odes of ethics", italienische "codice morale“, französische „déontologie“.

3. In Wirklichkeit sind gewisse Berufsvereinigungen katholischer Nationen nicht konfessionell, a) weil der Grundsatz der Religionsfreiheit erfordert, daß sie für jedwede eventuelle nicht-katholische Berufskraft offenstehen; b) weil sie nicht daran interessiert sind, ihren konfessionellen Charakter $\mathrm{zu}$ unterstreichen. Dessen ungeachtet sind sie in ihrer ganzen Tätigkeit von einer katholischen Weltanschauung durchzogen.

4. Hier ist eine mögliche $Z$ weideutigkeit auszuschalten. Geistliche können, genau wie Laien, eine Berufsausbildung genießen und die daraus erwachsende Funktion ausüben. Wer könnte dies bezweifeln? Ein Bischof, eine Bischofskonferenz, eine Religiosenkongregation oder eine andere kirchliche Einrichtung können auch nicht nur Schulen für die seelsorgliche Ausbildung im Gebrauch der Massenmedien, sondern echte Berufsschulen auf dem ganzen Gebiet der Informationswissenschaft und -technik gründen und leiten. Wer könnte dies bezweifeln? Zuweilen werden sie es sogar tun müssen, weil die Kirche jahrhundertelang auf dem Gebiet des Unterrichts eine Pionierarbeit geleistet hat; und die jungen Nationen brauchen Journalistenschulen, die von anderen nicht gegründet werden oder die nicht in ausreichender Zahl oder Qualität existieren. Es handelt sich hierbei um die Ausübung einer Freiheit - nämlich der Freiheit, einen Beruf auszuüben und Unterrichtszentren zu gründen -, auf die jeder Mensch ein Recht hat, und von einer subsidiären Pflicht; nicht um besondere Rechte und Pflichten, die in der Pastoralaufgabe begründet sind. Es gibt also keinen Anlaß zur Kompetenzverwechslung.

5. So ist z. B. für die UCIP keine Andeutung auf deren Gegenwart bei den internationalen Einriditungen wie UNO und UNESCO, oder auf deren Dialog mit den nicht-katholischen Berufsorganisationen zu finden; nichts über die ständige Tätigkeit im Sinne einer Koordinierung der katholischen Berufskräfte in den neutralen Medien, die zu einer der wichtigsten der UCIP wird; nichts über die überkonfessionellen Kontakte oder über die Beziehungen mit den internationalen katholischen Organisationen außerhalb der Massenmedien.

6. „Assicureranno alle organizzazioni internazionali l'aiuto economico necessario per svolgere il loro compito"; „assureront à ces Organisations catholiques internationales l'appui financier nécessaire pour accomplir leur mission“; „proporcionarán los medios económicos necesarios para que realicen sus objetivos".

\section{S U M M A R Y}

The Pastoral Instruction "Communio et Progressio", according to the author, gives certain principles regarding the relationship between the professional Catholic Organisations for Social Communications and the Ecclesiastical Centres set up in this field by the hierarchy. Using the example of the International Press Association (UCIP) he shows how the freedom of these professional organisations is respected. On the other hand, the Church cannot be disinterested with regard to its influencing Communications activities of Catholic journalists. It goes without saying that there exists a difference between the organisations of the Church which are dependant upon the hierarchy and the professional organisations within the Church. From this point of view the author comes to some important conclusions. 